Mycopathologia vol. 61, 1: pp. 27-33, 1977

\title{
COMPARATIVE RECOVERIES OF AIRBORNE FUNGUS SPORES BY VIABLE AND NON-VIABLE MODES OF VOLUMETRIC COLLECTION
}

\author{
Harriet P. BURGE, J. R. BOISE, J. A. RUTHERFORD \& W. R. SOLOMON \\ Section of Allergy, Department of Internal Medicine, and Department of Biostatistics, University of Michigan, Ann Arbor, \\ Michigan, USA
}

\section{Summary}

The suitability of viable and non-viable volumetric collectors as prevalence indicators for potentially allergenic airborne fungi was studied during 124 paired exposures of the Burkard (Hirst) spore trap and a modified, windoriented Andersen sampler. Overall, viable recoveries of several Cladosporium form species varied directly with microscopic spore counts ( $\mathrm{p} \leq 0.0001$ ). However, as spore levels rose, culture plate data progressively underestimated prevailing concentrations (recoveries falling below $5 \%$ at levels above 500 spores $/ \mathrm{M}^{3}$ ). Viable collections yielded low estimates of prevalence $(20-40 \%)$ even at modest Cladosporium levels ( $<100$ spores $/ \mathrm{M}^{3}$ ) and substantially understated the abundance and regularity in air of several additional taxa. Spores typical of Penicillium and Aspergillus form species were not sought in spore trap deposits. Careful examination of these failed to reveal typical arthrospores or Fusarium macrospores despite substantial recoveries of corresponding growth in culture. Correlations in the occurrence patterns of arthrospore-forming and non-sporulating colonies with those of Coprinus and 'other basidiospores' (excluding Ganoderma) were noted.

\section{Introduction}

Volumetric samplers of determinate efficiency, which permit viable or non-viable collections of many air spora components, presently are available. However, even when interest is confined to the most prevalent deuteromycete taxa, neither sampling approach alone is adequate. Both

\footnotetext{
* This work was supported by Research Grant AI-10181 from the National Institute of Allergy \& Infectious Diseases, National Institutes of Health, United States Public Health Service.
}

modes of recovery contribute uniquely to surveys of potential aeroallergens, although the limitations of data derived solely from cultures or from microscopic dust deposits often are accepted arbitrarily. The merits of these complementary approaches have been summarized (6) and recovery spectra described $(1,2,4,16)$. In general 'non-viable' techniques require only that particles have distinctive form, while germination and growth potential, which govern viable recoveries, are multifactorial variables and often defy accurate prediction. These uncertainties, coupled with traditional reliance upon cultural sampling methods mandate a comparison of available collectors as prevalence indicators for common imperfect fungi. Accordingly, we have studied 'viable' and 'nonviable' recoveries of suitable particles by two major volumetric devices (viz., the Andersen sampler and Burkard trap) under field conditions.

\section{Materials and methods}

Sampling was carried out on the roof of the Univ of Michigan's Kresge Medical Research Bldg., 50 above grade, in Ann Arbor. There is no parapet, and the site is unobstructed to the North, South and West, although a low elevator housing is present ca. $15^{\prime}$ to the East. Simultaneous particle collections were made between 08.30 and $09.30 \mathrm{hrs}$ on weekdays under a variety of weather conditions during the period from April 1973 to June 1974; as a result, a total of 124 paired samples was available for analysis.

The Burkard (Hirst) automatic volumetric spore trap (Burkard Mfg. Co., Ltd., Rickmansworth, Herts, England) is a continuously operating, wind-oriented particle sampler. The device has collection characteristics similar to a previously described Hirst trap (7) with a 10 liter/minute 
throughout and $2 \times 14 \mathrm{~mm}$ intake orifice. Particles entering the Burkard trap impinge on a slowly rotating drum covered with $19 \mathrm{~mm}$ wide Melenex ${ }^{(\mathbf{R})}$ tape coated (in the present study) with Lubriseal ${ }^{(R)}$ grease (A. H. Thomas Co., Philadelphia, $\mathrm{Pa}$.) as a surface adhesive. Initial calibration indicated that the collection surface advanced at $2.05 \mathrm{~mm} /$ $\mathrm{hr}(=49.2 \mathrm{~mm} /$ day $)$ prompting us to replace the drum every six days, when, in addition, the inflow rate was checked with a specially adapted rotameter. After removal from the drum the ca. $295.2 \mathrm{~mm}$ long strip of particlebearing tape was cut into $24 \mathrm{hr}$ segments; each was then mounted on a $1 \times 3$ inch microslide in hard glycerine jelly (8).

Particle counts were made at $312.5 \mathrm{x}$ from $50-60$ fields, (each 464 micrometers in diameter) inspected, using a graduated mechanical stage, in a traverse corresponding to $09.00 \mathrm{hrs}$. The following distinctive types were enumerated: smooth Cladosporium spores (designated ' $\mathrm{B}_{\text {clar }}$ '), rough Cladosporium spores (designated ' $\mathrm{B}_{\text {herb }}$ ') and spores typical of Alternaria, Epicoccum, Fusarium, Arthrinium, Pithomyces, Nigrospora, Torula, Botrytis and Helminthosporium-Drechslera species. Field counts for each particle type were converted to units/meter ${ }^{3}$ of air sampled by dividing by $0.003528 \mathrm{M}^{3}$ - the volume of air theoretically contributing particles to one microscopic field.

The Andersen (model 0101) sampler (2000 Inc., Atlanta, Ga.), in which $1 \mathrm{ft}^{3}(28.31$.)/min. of particle-bearing air is impinged directly upon semi-solid growth media, provided viable recoveries (3). For this study, the sampler was windoriented on the upper surface of the Burkard trap so that both intake orifices were aligned, as shown in Fig. 1, and

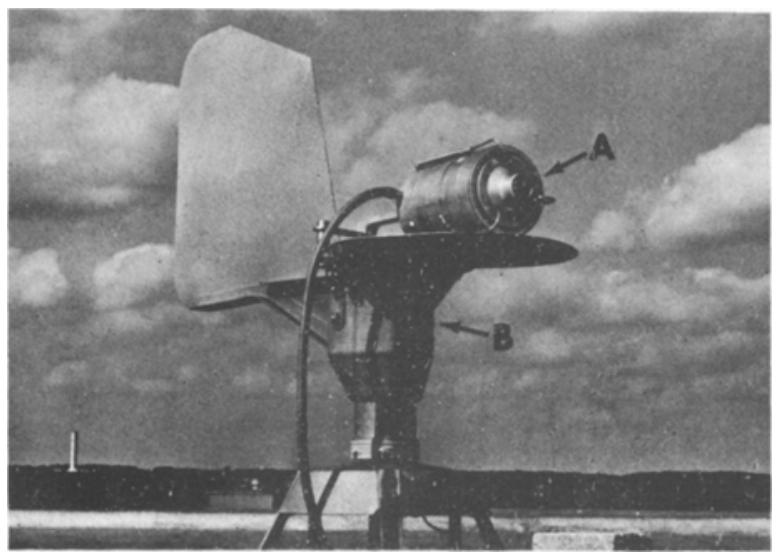

Fig. 1. The Burkard trap and the Andersen sampler on the roof of the Kresge Building. $A \rightarrow$ refers to the orifice of the Andersen sampler. $B \rightarrow$ refers to the orifice of the Burkard trap. ca. $8 \mathrm{~cm}$ apart. Malt extract agar (malt extract $32 \mathrm{gm}$, Bacto agar $16 \mathrm{gm}$, yeast extract $1 \mathrm{gm}$ and distilled water 1 liter) was used exclusively in carefully measured portions contained in special flat-bottomed Petri dishes. Single culture plates were placed beneath the sixth stage of the sampler to obtain collections integrated with respect to particle size. Carefully timed sampling periods of 30 second to ten minute duration, depending on prevailing spore levels, were employed to prevent overloading of the agar surface. Following exposure, the plates were incubated in high humidity at $22^{\circ} \mathrm{C}$ for seven days and all growth examined microscopically. Cladosporium form species were determined using the criteria of Ellis (5); other colonial types were identified only to form genus. Colony counts were corrected for the effect of multiple impactions at one point using Andersen's nomogram (3), and concentrations were calculated using the equation:

particles $/ \mathrm{M}^{3}=\frac{\text { total colonies }}{\text { sampling time (min) }} \times \frac{35.3 \mathrm{ft}^{3}}{1 \mathrm{~meter}^{3}} \times \frac{1 \mathrm{~min}}{1 \mathrm{ft}^{3}}$

To facilitate comparison, data for Cladosporium cladosporioides and C. sphaerospermum were grouped as ' $\mathrm{A}_{\mathrm{clad}}$ ' while those for $C$. herbarum and C. macrocarpum were combined and designated ' $\mathrm{A}_{\text {herb }}$ '. Recoveries of other Cladosporium form species were negligible. After collation of these data, Spearman rank order correlations and graphic comparisons of Cladosporium recoveries were done with the MIDAS statistical package, Statistical Research Laboratory, Univ. of Michigan on the Amdahl 470 computer.

\section{Results}

Andersen cultural recoveries, expressed as spores $/ \mathrm{M}^{3}$ of air sampled for Cladosporium cladosporioides plus $C$. sphaerospermum $\left(\mathrm{A}_{\text {clad }}\right)$ were directly correlated with levels of smooth Cladosporium spores $\left(\mathrm{B}_{\text {clad }}\right)$ calculated from Burkard trap collections. Similarly, Andersen data for $C$. herbarum plus $C$. macrocarpum $\left(\mathrm{A}_{\text {herb }}\right.$ ) varied directly with Burkard recoveries of rough Cladosporium spores $\left(\mathbf{B}_{\text {herb }}\right)$. Total Cladosporium counts from viable and non-viable samples respectivelv, $\left(A_{\text {sum }}\right.$ and $B_{\text {sum }}$ ) were similarly correlated (Spearman rartk order correlation, $\mathrm{p}<0.0001$ for the three comparisons). Daily Burkard and Andersen counts for 'clad', 'herb', and 'sum' categories are compared in Fig. 2. Although considered in the rank order correlations, the nine days of highest counts have been excluded from the graphs to allow better visualization of days with more 


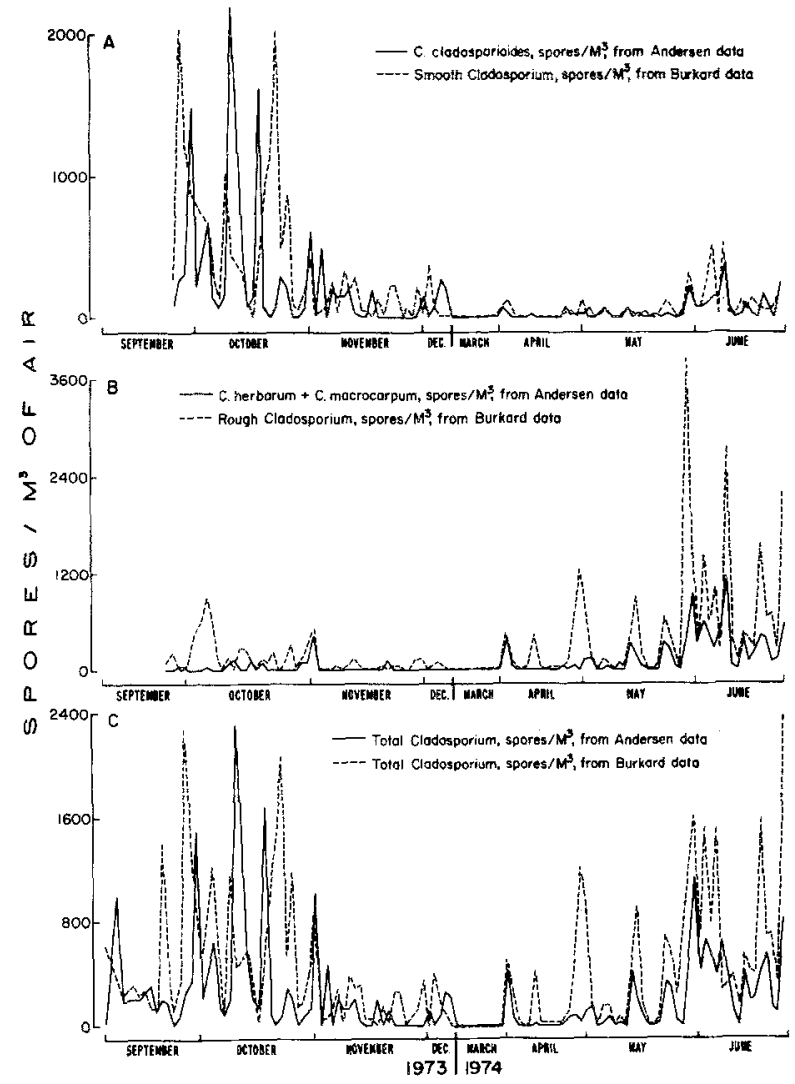

Fig. 2. Comparative recoveries of Cladosporium spores with the Andersen sampler (-) and the Burkard trap (---); top panel smooth Cladosporium spores (C. cladosporioides), center panel rough Cludosporium spores ( $C$. herbarum $+C$. macrocarpum), lower panel total Cladosporium recoveries.

typical concentrations. On each of the nine excluded days, levels calculated from Burkard counts far exceeded those from Andersen sampler recoveries.

Figure 3 shows the relationships between $B_{\text {clad, }}, B_{\text {herb }}$ and $B_{\text {sum }}$ and the recovery differences (i.e., $B_{\text {clar }}$ minus $A_{\text {lad }}$, $B_{\text {herb }}$ minus $A_{\text {herb }}$, and $B_{\text {sum }}$ minus $A_{\text {sum }}$, respectively) between samplers. Also compared are $B_{\text {clad }}, B_{\text {herb }}$ and $B_{\text {sum }}$ and the percentages $A_{\text {clad }} / B_{\text {clad }} \times 100, A_{\text {herb }} / B_{\text {herb }} \times 100$ and $A_{\text {sum }} / B_{\text {sum }} \times 100$, respectively. These data indicate that the reliability of the Andersen sampler as an indicator of Cladosporium spore levels declines sharply as higher spore concentrations are reached (recoveries falling below $5 \%$ at levels above 500 spores $\left./ \mathrm{M}^{3}\right)$. Furthermore, even at low particle concentrations $\left(<100\right.$ spores $\left./ \mathrm{M}^{3}\right)$ the Andersen sampler, (when employed in this fashion,) underestimates prevailing Cladosporium levels by $60-80 \%$.

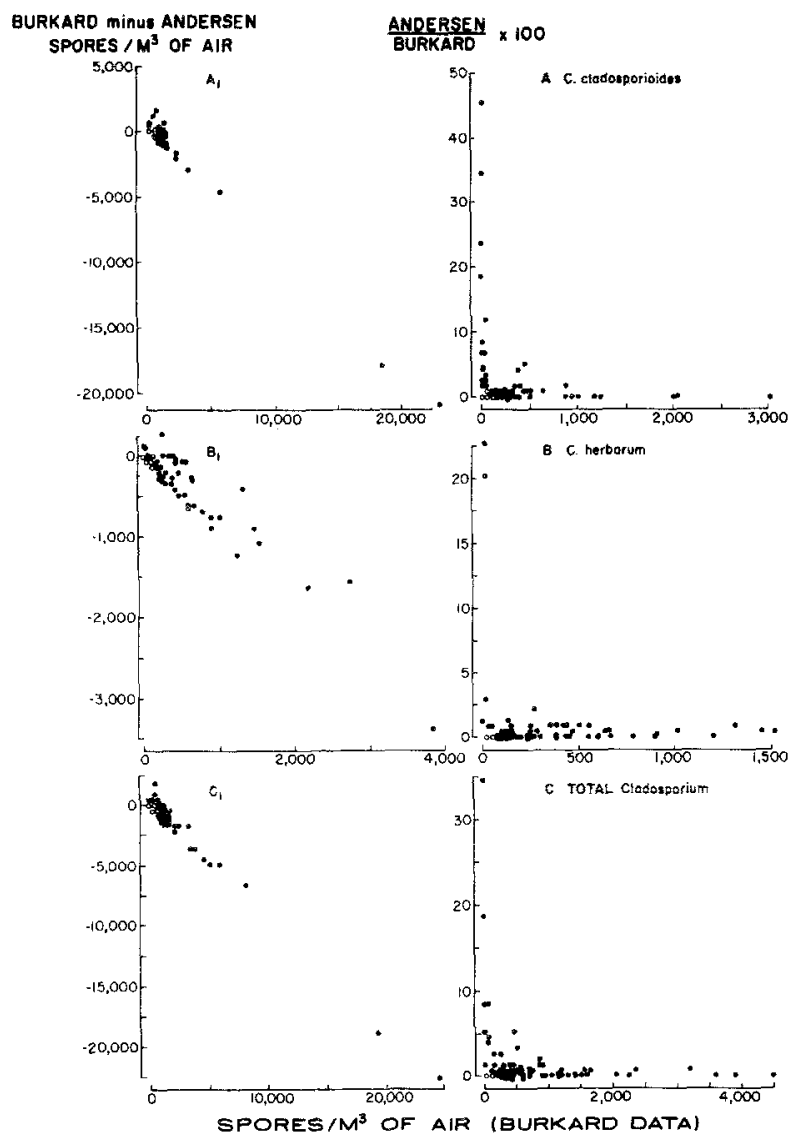

Fig. 3. The Burkard-Andersen-difference (Ieft column) and the Andersen/Burkard percentage (right column) plotted against spores/ $\mathbf{M}^{3}$ of air from Burkard data for smooth Cladosporium spores (top panel), rough Cladosporium spores (center panel) and total Cladosporium spores (lower panel).

Table 1 lists, for all taxa studied, the consistency of recovery (days of recovery/total sampling days $\times 100$ ) from Andersen and Burkard samples, as well as the mean, standard error of the mean, median, mode, and range of indicated concentrations from Burkard (or Andersen) data. For all types except Fusarium and Penicillium/ Aspergillus, both frequency of recovery and levels of recovery were higher for Burkard samples. Fusarium macrospores were not seen on Burkard trap deposits in any of the several thousand fields examined. Microspores typical of Fusarium form species cannot be identified as such, and spores of their perfect stages (e.g., Gibberella species) are not reliably separable from those of other ascomycete taxa by microscopic characters alone.

The 'Penicillium/Aspergillus' category includes additional taxa producing similar spores, although ninety 
Table 1. Comparative frequencies of recovery and prevalence levels for the taxa studied. Ranges and measures of central tendency calculated from Andersen data are shown in parentheses. Unless otherwise stated, all other data are derived from Burkard trap recoveries

\begin{tabular}{|c|c|c|c|c|c|c|c|}
\hline & \multirow{2}{*}{\multicolumn{2}{|c|}{$\begin{array}{l}\text { Frequency of recovery } \\
\frac{\text { Days recovered }}{\text { total days }} \times 100\end{array}$}} & \multicolumn{5}{|c|}{ Spores $/ M^{3}$ of air } \\
\hline & & & Mean & SEM & Median & Mode & Range \\
\hline & Andersen & Burkard & & & & & \\
\hline Cladosporium & 68 & 89 & 1238 & 255 & 399 & 34 & $6-24,000$ \\
\hline Penicillium/Aspergillus & 74 & (0) & $(86)$ & (104) & (35) & $(70)$ & $(3-848)$ \\
\hline Alternaria & 19 & 62 & 32 & 4 & 17 & 6 & $5-300$ \\
\hline Epicoccum & 4 & 51 & 36 & 4 & 21 & 6 & $5-245$ \\
\hline Botrytis & 1 & 28 & 14 & 1.5 & 11 & 6 & $5-67$ \\
\hline Pithomyces & 1 & 18 & 18 & 3 & 11 & 6 & $5-64$ \\
\hline Helminth./Drechslera & 0 & 18 & 8 & 1 & 6 & 5 & $5-18$ \\
\hline Torula & 0 & 16 & 21 & 3 & 14 & 5 & $5-69$ \\
\hline Arthrinium & 3 & 10 & 39 & 8 & 25 & 23 & $5-131$ \\
\hline Fusarium & 7 & $(0)$ & $(56)$ & (8) & $(53)$ & $(70)$ & $(17-140)$ \\
\hline
\end{tabular}

percent of the recoveries included here were Penicillium form species. The origins of small, spherical spores cannot be determined reliably from Burkard trap samples, and their distinction from other globular particles often is difficult.

Arthrospores are asexual spores, which appear quite distinctive when mounted on Lubriseal-coated Melenex tape in glycerine jelly. However, in spite of careful and repeated searches on days when Andersen recoveries of arthrospore-forming colonies were heavy, we were unable to identify these particles on Burkard trap deposits. This discrepancy may indicate that arthrospores are produced in culture by fungi derived from sexual airborne spores just as sexual spores may produce nonsporulating colonies in culture. An attempt was made, therefore, to correlate Burkard counts of Ganoderma, Coprinus and 'other basidiospores' individually with arthrospores and nonsporulating recoveries from the Andersen sampler; the resulting data are presented in Table 2. Coprinus spore levels were approximately equal for days of 'recovery' and 'no recovery' of both arthrospores and nonsporulating colonies. Coprinus species, therefore, cannot be overlooked as sources of nonsporulating growth on malt agar or of arthrospore-forming recoveries. One nonsporulating colony eventually produced a Coprinus fruiting body

Table 2. Comparison of Burkard levels of three basidiospore categories with the presence or absence, on Andersen samples, of arthrospore bearing and nonsporulating (NS) colonies

\begin{tabular}{|c|c|c|c|c|c|}
\hline & & & Burk & rd levels & (spores $\left./ M^{3}\right)$ \\
\hline & & & Ganoderma & Coprinus & $\begin{array}{c}\text { Other } \\
\text { Basidiospores }\end{array}$ \\
\hline 照 & & Days & Mean SEM & Mean SEM & Mean SEM \\
\hline$\sum_{0}^{d}$ & Arthrospores present & 12 & $490 \pm 120$ & $455 \pm 104$ & $360 \pm 87$ \\
\hline$\stackrel{0}{\infty}$ & Arthrospores absent & 14 & $653 \pm 159$ & $518 \pm 104$ & $198 \pm 36$ \\
\hline 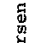 & NS colonies present & 18 & $334 \pm 93$ & $510 \pm 115$ & $318 \pm 88$ \\
\hline$\stackrel{0}{g}$ & Ns colonies absent & 8 & $1072 \pm 334$ & $407 \pm 205$ & $173 \pm 61$ \\
\hline
\end{tabular}


adding support to this conclusion. 'Other basidiospores' were found to be more prevalent on days presenting arthrospore-forming and nonsporulating colonies than on days without such recoveries. However, Ganoderma mean spore levels appeared to be negatively correlated with the presence, in culture, of both arthrospore sources and nonsporulating growth.

\section{Discussion}

These data confirm the validity of both viable and nonviable volumetric collections as indicators of prevalence trends for selected spore types. The comparative accuracy of the two methods employed cannot be judged without recourse to an absolute isokinetic standard. However, the consistently better representation of all particle types in Burkard trap collections suggests that they more clearly reflect true variations in levels of air spora. Possible bases for differences in performance between these devices include disparities in free air collection efficiency as well, of course, as the complex variable of germination potential to which viable recoveries are subject.

By aligning the two sampler orifices, equivalent, gustresponsive, wind orientation was assured; however the intake airspeeds respectively 21.4 and $3.1 \mathrm{~km} / \mathrm{hr}$ for Burkard trap and Andersen samples differed substantially. Since the free stream velocity between 08.30 and 09.30 hours commonly was between these values, anisokinetic conditions must have been the rule. A factitious lowering of Burkard trap recoveries should result from these airspeed inequalities while excessive particle capture, leading to overestimates of true prevalence, would be predicted for the Andersen sampler (11). Such bias may have reduced observed differences in particle capture by the two devices. However, this effect was not discernible when wind speed and the ratio between Andersen and Burkard recoveries for total Cladosporium were examined using rank order correlations. Similar comparisons for substantially larger or smaller particle types might have revealed greater differences in capture efficiency by these devices with extremes of wind speed. Anisokinetic sampling conditions are known to impose the smallest errors on the collection of particles of ca. 20 micrometers diameter (17), a size approximated by spores or spore aggregates of several of the deuteromycete taxa considered.

'Slippage', or passage of aspirated (multimicronic) particles completely through the sampler (3), appears to be low for both devices, although published data are wanting for the Burkard trap. The risk of such losses is increased theoretically by using only a single plate in the Andersen sampler but was mitigated by empirically reducing collection time. Some increase in the relative sampling error associated with Andersen data must have occurred as a result. However, remarkably similar estimates of particle prevalence have been derived from single plate collections and (standard) six plate arrays when appropriate sampling times were employed (13).

Environmental variables which affect spore hydration and charge were not studied. We suspect, however, that hydration would have relatively little effect on the differential behavior of the biological particles studied here.

The major variable confronting any viable particle sampling method is germination and growth potential. This complex attribute encompasses viability, characteristics of spore dormancy, nutritional requirements for germination and growth, and intra- and interspecific competitive inhibition before and after germination. Viability, in absolute terms, depends on sets of factors that include environmental (temperature, humidity, insolation, degree and kind of atmospheric pollution); mechanical (impaction resistance); and biological (inherent viability at time of production, effects of aging, etc.,) variables. However, under the conditions of this study, viability is not easily distinguished from other aspects of germination potential.

Spore dormancy has been extensively reviewed by Sussman $(14,15)$. His discussions emphasize that spores of diverse fungi require some exogenous treatment to break dormancy and initiate germination; at least one species of Coprinus, for example, requires a combination of heat and chemical activation. Unfortunately, none of the taxa considered in this study have been examined with respect to dormancy and requirements for its termination. Appropriate activation conditions may be assumed for Alternaria, Cladosporium and possibly Epicoccum species. However, further study will be necessary to demonstrate that the activation requirements of remaining taxa have been met.

Nutritional requirements for germination can be considered as one essential facet of the activation process. Once the spore has germinated, however, growth must occur for a positive Andersen recovery. Many fungi have extremely specific nutritional requirements for growth which not only affect absolute ability to grow but also growth rate. We chose for study only taxa known to accept cultivation on malt extract agar, and restricted our studies to this single medium. Since we could not optimize conditions for 
all taxa, several additional types undoubtedly were lost due to insufficient growth for recognition or to overgrowth by more versatile fungi.

Studies of competition among fungi in culture have been confined to a very few taxa and have been restricted to the investigation of self-inhibition (9). None of the taxa considered in this study have been examined with respect to either self- or cross-inhibition. However the data presented in Fig. 3, indicating that the percentage of Cladosporium recoveries on Andersen plates declines with increased Burkard Cladosporium counts, could be explained on the basis of self-inhibition affecting either germination or growth. The low frequency of recovery of other taxa listed in Table 2 on Andersen plates compared to Burkard recoveries may also reflect either self- or crossinhibition,

Viable particle sampling often is essential, especially for the many fungi that lack microscopically distinctive spores. This group includes the ubiquitous form species of Aspergillus, Penicillium and related genera. It is, in fact, difficult, at times, to distinguish between the small globular spores of the fungi and inorganic ash particles in dust deposits. Yeast and bacteria are other prominent groups which require colony counts for enumeration, and the infective potential of a spore cloud can be determined only by culture methods. Viable collections can contribute substantially to studies of spore germination potential. However, with recognizable particles, the method of Pathak \& Pady (12) for determining germination percentage for particle samples captured on silicone-coated slides seems preferable since it eliminates problems of unsatisfied growth requirements and competitive inhibition during the growth phase.

The abundant asco- and basidiospores recovered in particle deposits are only very rarely represented by appropriate perfect fruiting stages on laboratory culture media (16). Further cultural surveys using, especially, natural substrates are clearly justified. However, in three years of extensive sampling with malt extract agar, we have seen only a single Coprinus fruiting body in culture; ascocarps have also, at best, occurred rarely.

Attempts described above to correlate athrospore-forming and sterile colonies with basidiospore levels were based on the hypothesis that certain of these sexual spores are capable of germination and recognizable growth in culture. The data presented admit this interpretation, and the association of arthrospores with stages of certain woodrotting basidiomycetes (10) suggests that further correlations may be fruitful. However, progress in this area will require an improved understanding of the potential of diverse basidiospores for growth in culture as well as improved recognition criteria for these particles. The possibility that specific ascospore types similarly may lead to identifiable growth in culture also is worthy of study.

Any extension of these data to other non-viable or viable particle collection methods must remain speculative. However, similar needs related to germination and growth potential confront all cultural methods. Likewise, spore appearance imposes natural limitations on any system of microscopicidentification. Where comprehensive estimates of airborne fungus prevalence are sought, the complementarity of the two volumetric approaches as well as their intrinsic limitations must be appreciated. This study emphasizes the probability that cultural recoveries will tend, in any case, to understate actual prevalence and reminds us of the several uncontrollable variables that affect viable collections.

\section{References}

1. Ackermann, H.-W. 1968. La recherche des champignons de l'air par culture depuis 1940. Rev. Canadienne de Biologie 27: 61-68.

2. Adams, K. F. 1964. Year to year variation in the fungus spore content of the atmosphere. Acta Allergologica 19: $11-50$.

3. Andersen, A. A. 1958. A new sampler for the collection, sizing and enumeration of viable airborne particles. $J$. Bacteriol. 76: 471-484.

4. Chatterjee, J. \& F. E. Hargreave. 1974. Atmospheric pollen and fungal spores in Hamilton in 1972 estimated by the Hirst automatic volumetric spore trap. Canad. M. Assn. J. 110: 659-663.

5. Ellis, M. B. 1971. Dematiaceous Hymophycetes. Commonwealth Agricultural Bureaux, Kew, U.K.

6. Gregory, P. H. 1973. Microbiology of the Atmosphere. John Wiley \& Sons, New York, 2nd ed.

7. Hirst, J. M. 1952. An automatic volumetric spore trap. Ann. Appl. Biol. 39: 257-265.

8. Johansen, D. A. 1940. Plant microtechnique. McGraw-Hill, New York.

9. Macko, V., et al. 1976. Self inhibitors of fungal spore germination. In: The Fungal Spore. D. J. Weber \& W. M. Hess (eds.). Wiley-Interscience, New York. pp. 73-100.

10. Nobles, M. K. 1971. Cultural characters as a guide to the taxonomy of the Polyporaceae. In: Evolution in the Higher Basidiomycetes. R. H. Peterson (ed.). University of Tennessee Press, Knoxville, pp. 169-192.

11. Ogden, E. C., G. S. Raynor, J. V. Hayes, D. M. Lewis \& J, H. Haines. 1974. Manual for Sampling Airborn Pollen. Hafner Press, New York. pp. 45-47. 
12. Pathak, V. K. \& S. M. Pady. 1965. Numbers and viability of certain airborne fungus spores. Mycologia 47: 301-310.

13. Solomon, W. R. \& J. A. Gilliam. 1970. A simplified application of the Andersen sampler to the study of airborne fungus particles. J. Allergy 45: 1-13.

14. Sussman, A. S. 1976. Activation of fungal spore germination. In: The Fungal Spore. D. J. Weber \& W. M. Hess (eds.). Wiley-Interscience, New York. pp. 101-139.

15. Sussman, A. S. \& H. D. Halvorson. 1966. Spores: their dormancy and germination. Harper and Row, New York.

16. von Arx, J. A. 1970. The Genera of Fungi Sporulating in Pure Culture. J. Cramer, Lehre, W. Germany.

17. Watson, H. H. 1954. Errors due to anisokinetic sampling of aerosols. Am. Ind. Hyg. Assn. Quart. 15: 21-25. 